\title{
Sonority projection effect in French: A signal detection theory approach
}

\author{
Anahita Basirat ${ }^{1, *}$, Cédric Patin ${ }^{2}$, Jérémie Jozefowiez ${ }^{1}$ \\ 1. Univ. Lille, CNRS, UMR 9193 - SCALab - Sciences Cognitives et Sciences Affectives, F-59000 \\ Lille, France \\ 2. Univ. Lille, CNRS, UMR 8163 - STL -Savoirs, Textes, Langage, F-59000, Lille, France \\ *: Corresponding author \\ Email: anahita.basirat@univ-lille.fr
}

\begin{abstract}
Focusing on the Sonority Sequencing Principle, we investigated to what extent adult native speakers of French are sensitive to sonority-related constraints compared to lexical attestedness. In a non-word acceptability task, participants were asked to rate the acceptability of three types of non-words using a 6-point scale: non-words with attested sonority rising onset, non-words with unattested sonority rising onset and non-words with unattested sonority falling onset. Data analysis was done using the signal detection theory approach to measure sensitivity of participants to lexical attestedness and to phonological well-formedness (i.e. respecting or violating the Sonority Sequencing Principle). The results showed that speakers distinguished well-formed and ill-formed forms even when lexical attestedness was controlled for. This is consistent with previous findings on sonority projection effects. Participants were more sensitive to lexical attestedness than phonological well-formedness. Future research using computational models should investigate the mechanisms which could account for these findings, namely whether a similar result would be obtained without including any assumption about the Sonority Sequencing Principle in these models.
\end{abstract}

\section{Keywords}

Phonological constraints; Sonority; Lexicon; Signal detection theory 


\section{Introduction}

Building upon "sonority law" (Sievers, 1876) and typological overviews such as that of Greenberg (1978), Clements (1990) introduced the Sonority Sequencing Principle (henceforth SSP), which states that "between any member of a syllable and the syllable peak, only sounds of higher sonority rank are permitted" (Clements, 1990, p. 285). ${ }^{1}$ Hence, an onset cluster such as $\mathrm{pl}$ can precede a vowel nucleus, since the sonority of the liquid [I] is higher than that of the stop [p], while Ip will be ruled out. Conversely, Ip is a suitable coda cluster according to the SSP, while a coda cluster $\mathrm{pl}$ should be dismissed. Several authors have attempted to explain such trends (for a review, see Parker, 2017). The main issue is to understand whether the constraints concerning the syllable structure emerge from the statistical properties of languages (e.g. syllable frequency in the lexicon) or whether they reflect a formal phonological rule.

To address this question, a classical approach is to examine how phonological constraints, such as SSP, are transferred to novel forms. The rationale is as follows. If phonological constraints are independent of the learned examples in the lexicon, speakers should apply these rules to new linguistic material. Otherwise, if phonological constraints emerge from the examples in the lexicon, speakers will probably process new forms differently from lexically attested forms. Using this rationale, several experimental studies compared the acceptability of non-words with syllables with CC clusters (C: consonant) respecting or not the SSP (e.g. Greenberg \& Jenkins, 1964; Albright, 2009; Daland et al., 2011; Hayes \& White, 2013). Speakers considered non-words beginning with rising sonority clusters (e.g. onset $p l$ ) more acceptable as potential new words than those beginning with falling sonority clusters (e.g. onset Ip), even when the degree of lexical attestedness of the clusters were controlled for. Such effects, known as sonority projection effects (Daland et al., 2011), exist in various languages and seem to be distinct from sensorimotor constraints (for a review, see Berent, 2017). They

$1 \mathrm{SSP}$ has been criticized for its failure to predict reversals such as s+stop clusters or the rarity of sequences such as $t l$, and its supposed universality has been questioned. These questions are beyond the scope of this paper - see Henke, Kaisse \& Wright (2012) for an overview. 
are thought to demonstrate the existence of a formal phonological rule concerning SSP (Berent, Steriade, Lennertz, \& Vaknin, 2007).

However, the above-mentioned interpretation has been challenged by modeling studies suggesting that a more modest assumption about SSP is sufficient to obtain sonority projection effects (e.g. Hayes, 2011). For instance, experimental data can be obtained if the frequency of features in the lexicon is taken into account, without assuming the SSP in the model. For example, although [Ib] and [tl] onsets are equally unattested in English words, they are not equally infrequent featurally as there are syllable onsets in English which are featurally similar to [tl] but not to [lb]. Therefore, native speakers of English may show sonority projection effects only by extracting and generalizing these featural cues without pre-existing SSP (Daland et al., 2011; Van de Vijver \& Baer-Henney, 2012, for German). The ability to generalize from the inventory of existing sonorityrespecting clusters seems to be one of the key mechanisms in such models to generate sonority projection effects without implementing the SSP as a formal phonological rule (e.g. Hayes, 2011, see also Bárkányi, 2011, on the role of relative probability of different combinations of natural classes).

Although these studies show that sonority projection effects can be predicted by lexicon statistics (but see Jarosz (2017) for another pattern in Polish), this does not imply that non-lexical knowledge is not taken into account while processing novel forms. For example, using a series of phonological constraints which were learnable from the lexicon, Hayes and White (2013) observed that speakers did not solely use their lexiconbased knowledge but also relied on a set of constraints which were irrespective of the lexicon (e.g. to avoid articulatory difficulty and/or to maintain perceptual distance between contrasting forms). They posited that speakers have a "learning bias" and do not evaluate new forms only in terms of learned examples.

The current study aimed to better understand the interplay between the processing of lexical attestedness and SSP violations in French. Participants were asked to rate the acceptability of three types of CCVC non-words using a 6-point scale: non-words with attested sonority rising, non-words with unattested 
sonority rising and non-words with unattested sonority falling CC onsets. Hereafter, in accordance with the previous studies on the sonority projection effects (e.g. White \& Chiu, 2017; Ulbrich, Alday, Knaus, Orzechowska, \& Wiese, 2016), we refer to sonority rising clusters as phonological well-formed clusters and to sonority falling as phonological ill-formed ones. Thus, in our study, only well-formed non-words are "sonoritycompliant". In addition to classical analyses of rating scores, we used the signal detection theory approach (Macmillan \& Creelman, 1991). This approach is highly relevant as it allows the sensitivity of speakers to lexical attestedness and phonological well-formedness to be measured precisely without any response bias (see Huang \& Ferreira (2020) for a discussion about the application of this approach to acceptability judgment in linguistic tasks). We expected that participants would be sensitive to both lexical attestedness and phonological wellformedness but perhaps to a different degree.

\section{Methods}

\subsection{Participants}

Twenty-four (17 females) native French speakers participated in the study. They were recruited through our personal network or via an announcement. Most of them were students at the University of Lille. Their mean age was 23 years $(S D=2)$. Their mean number of years of education was $16(S D=1)$. All had self-reported normal or corrected-to-normal vision without any hearing or language problems. Their performance in auditory phonological discrimination and written lexical decision, assessed with standardized French batteries (respectively, Majerus et al., 2005; Macoir et al., 2016), were in the normal range. An additional individual was tested but excluded because their data could not be used in the analysis (see Data analysis). The study was conducted in accordance with the tenets of the Helsinki Declaration. Before testing, all participants gave their written informed consent.

\subsection{Material}


Three lists of 48 CCVC non-words were generated by concatenating a CC onset with a VC tail (e.g. bl + ar $\rightarrow$ blar) (Appendix A). The three lists differed in terms of the syllable onsets, which were (1) frequent in French with rising sonority, such as $d r$; (2) unattested or very rare in French with rising sonority, such as $t$, or (3) unattested or very rare in French with falling sonority, such as $v p$. The sonority profile of the onsets was calculated using Steriade (1982)'s scale. Their frequency was extracted from the Lexique database (New et al., 2004). Twelve VC tails were selected among possible tails in French. Each tail was used four times with four different clusters in each list (12 tails x 4 CC per list = 48 non-words per list). Non-words of the attested and well-formed list and those of the unattested and well-formed list were matched in terms of the sonority profile of their onset but were significantly different in terms of the frequency of their onset and the number of phonological and orthographic neighbors (Appendix B). Non-words of the unattested and well-formed list and those of the unattested and ill-formed list were matched in terms of the frequency of their onset and the number of phonological and orthographic neighbors but were significantly different in terms of the sonority profile of their onset (Appendix B). Each list constituted one experimental condition in our study (see below).

\subsection{Experimental design and procedure}

The experimental design was adapted from Daland et al. (2011). During the experiment, participants performed a non-word acceptability task on 144 non-words (48 non-words $x 3$ conditions). For each non-word presented in written form, participants were asked to indicate the likelihood that it would become a new word in French. The possible responses were 1 (unlikely), 2, 3, 4, 5 and 6 (likely). Stimuli were presented on the screen and participants scored them by clicking on a visual Likert scale. The order of presentation was fully randomized. Presentation and response collection were performed using Octave software and Psychtoolbox.

\subsection{Data Analysis}

We performed two types of analyses to examine how participants rated different non-words. The main approach was based on the signal detection theory (Macmillan \& Creelman, 1991), which is commonly used in 
the field of psychophysics (e.g. Jozefowiez, Gaudichon, Mekkass, \& Machado, 2018) and psycholinguistics (e.g. Sato et al., 2011) (see also Huang \& Ferreira, 2020). We also analyzed the data using a more classical approach to test the effect of Condition on participants' responses. To do so, the mean of ratings was calculated per participant and per condition. A repeated-measure ANOVA was conducted on these values with Condition as within-subject factor. The post-hoc comparisons were done using t-tests.

Regarding the signal detection theory approach, we performed a similar analysis as in Jozefowiez et al. (2018). For each participant and each list, the proportion of trials for which the participant provided the rating $R$ ( $R \in[1,2,3,4,5,6])$ was computed. These proportions were used to perform data analysis. Figure 1 shows the model on which the analysis relied. We assume that, when presented with a non-word, the participant's answer is based on the value of an internal decision variable $x$. There is a set $\left[\lambda_{1}, \lambda_{2}, \lambda_{3}, \lambda_{4}, \lambda_{5}\right]$ of critical values for the decision variable: If $x$ falls below $\lambda_{1}$, the participant provides the rating 1 ; if $x$ falls between $\lambda_{1}$ and $\lambda_{2}$, the participant provides the rating 2, etc. When presented with an unattested and ill-formed onset (referred to as UA-IF in the following equations) (respectively unattested and well-formed referred to as UA-WF, attested and well-formed referred to as A-WF onset), $x$ would be drawn from a Gaussian distribution with mean $\mu_{U A-I F}$ (respectively $\mu_{U A-W F}, \mu_{A-W F}$ ) and standard deviation $\sigma$. Without loss of generality, we can assume $\sigma=1$. Let $i$ be an integer between 1 and 6. $P_{U A-I F}(i)$ (respectively $P_{U A-W F}(i), P_{A-W F}(i)$ ) is the probability that the participant provides a rating between $i$ and 6 when presented with an unattested and illformed onset (respectively unattested and well-formed, attested and well-formed onset). Let $d^{\prime}(U A-I F, U A-W F)$ be the difference $\mu_{U A-I F}-\mu_{U A-W F}$. It is a measure of the ability of the participant to discriminate between non-words with unattested and ill-formed and unattested and well-formed onset, which is not affected by any response bias toward a specific rating the participant might have. It can be shown that

$$
d^{\prime}(U A-I F, U A-W F)=Z\left(P_{U A-I F}(i)\right)-Z\left(P_{U A-W F}(i)\right)
$$


Where $Z(x)$ is the inverse cumulative distribution of the standard normal distribution.

Likewise, $d^{\prime}(A-W F, U A-W F)$, i.e. $\mu_{A-W F}-\mu_{U A-W F}$, can be computed using Equation (2)

$$
d^{\prime}(A-W F, U A-W F)=Z\left(P_{A-W F}(i)\right)-Z\left(P_{U A-W F}(i)\right)
$$

Thus, obtaining a $d^{\prime}$ near 0 suggests that participants do not discriminate the corresponding lists and that they are not sensitive to the manipulated factor (i.e. attestedness or well-formedness).

Note that to calculate each $d^{\prime}$, i was set to a different value to satisfy two constraints. First, $Z(x)$ is not defined for 0 and 1 . Second, the higher the $P$ values, the better the estimate of $d^{\prime}$. Choosing different $i$ is not problematic because the value of $d^{\prime}$ is independent from the location of the response criteria. One participant was excluded as none of the values for $i$ allowed these two constraints to be satisfied. Table 2 provides an example of our analysis and shows the values of $i$, the portion of the data they induced, and the values of $P$ and $d$ ' computed from them for one of the participants. 


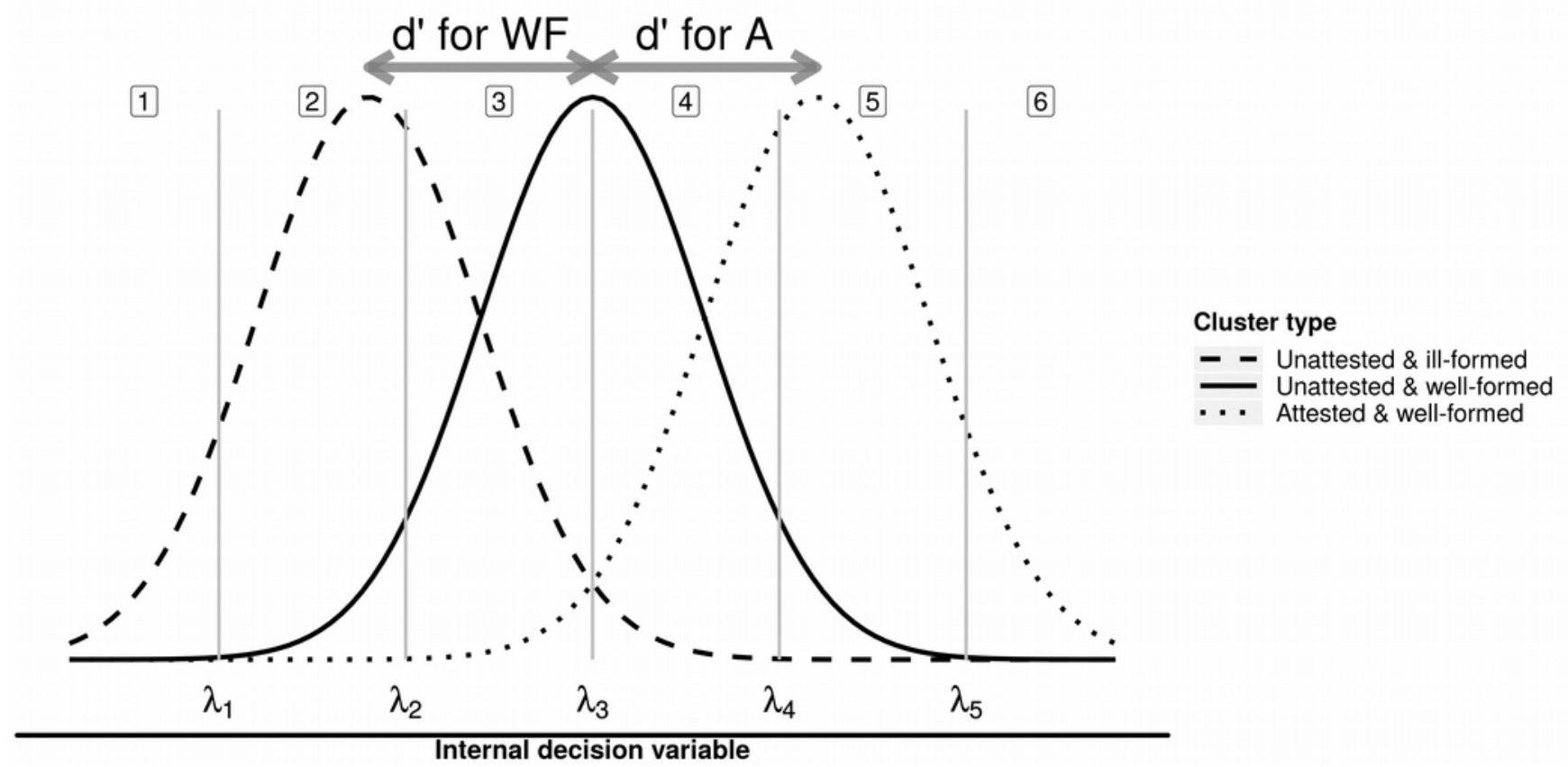

Figure 1: Signal detection theory model of non-word acceptability task. Each Gaussian curve shows distribution of acceptability for each list used in experiment. The acceptability continuum is divided by response criteria corresponding to participants' ratings (1: unlikely to 6: likely). d'for WF and d'for A reflect sensitivity to onset well-formedness and onset attestedness, respectively. 


\begin{tabular}{|c|c|c|c|c|c|c|c|c|c|c|c|c|c|c|c|}
\hline & \multicolumn{12}{|c|}{ Number of responses corresponding to 1 to 6} & $i$ & $P$ & $d^{\prime}$ \\
\hline & \multicolumn{6}{|c|}{ well-formed onset } & \multicolumn{6}{|c|}{ well-formed onset } & \multirow{3}{*}{4} & \multirow{3}{*}{$\begin{array}{c}P_{A-W F}=0.98 \\
P_{U A-W F}=0.06\end{array}$} & \multirow{3}{*}{3.6} \\
\hline \multirow{2}{*}{ Sensitivity to Attestedness } & 1 & 2 & 3 & 4 & 5 & 6 & 1 & 2 & 3 & 4 & 5 & 6 & & & \\
\hline & 0 & 0 & 0 & 1 & 11 & 36 & 7 & 23 & 11 & 4 & 2 & 1 & & & \\
\hline & \multicolumn{6}{|c|}{ ill-formed onset } & \multicolumn{6}{|c|}{ well-formed onset } & 3 & $P_{U A-I F}=0.13$ & -01 \\
\hline \multirow[t]{2}{*}{ Sensitivity to Well- } & 1 & 2 & 3 & 4 & 5 & 6 & 1 & 2 & 3 & 4 & 5 & 6 & & \multirow[t]{2}{*}{$P_{U A-W F}=0.15$} & \\
\hline & 24 & 12 & 6 & 6 & 0 & 0 & 7 & 23 & 11 & 4 & 2 & 1 & & & \\
\hline
\end{tabular}

Table 1: Example of the signal detection theory analysis used for one of the participants. See Data Analysis for

details about $i, P$ and $d$. Portion of data induced by $i$ is shown in gray.

\section{Results}

The data corresponding to our analyses are shown in Figure 2. The average responses were $5.16(95 \%$ Confidence Interval, C.I. $=[4.95$ 5.38] $), 2.28(95 \%$ C.I. $=[2.02$ 2.54]) and $1.52(95 \%$ C.I. $=[1.33$ 1.71 $])$ for attested and well-formed, unattested and well-formed and unattested and ill-formed conditions, respectively (see Figure 2A). The effect of Condition was significant $(F(2,46)=790.9, p<0.001)$. Pairwise comparisons showed that nonwords with attested and well-formed onset were rated higher than those with unattested and well-formed $(t(23)=29.23, p<0.001)$ and unattested and ill-formed $(t(23)=35.05, p<0.001)$ onsets. Non-words with unattested and well-formed onsets were also rated higher than those with unattested and ill-formed onsets $(t(23)=8.76, p<0.001)$

Figure $2 \mathrm{~B}$ shows the values of $\mathrm{d}$ '. The mean $\mathrm{d}^{\prime}$ for the analysis performed on unattested and well-formed and attested and well-formed conditions, reflecting sensitivity to attestedness, was $2.73(95 \%$ C.I. $=[2.51,2.95])$. The d' for the analysis performed on unattested and well-formed and unattested and ill-formed conditions, 
indicating sensitivity to well-formedness, was -0.99 (95\% C.I. $=[-1.21,-0.77])$. The difference between d' for attestedness and d' for well-formed was significant $(t(23)=30.70, p<0.001)$.

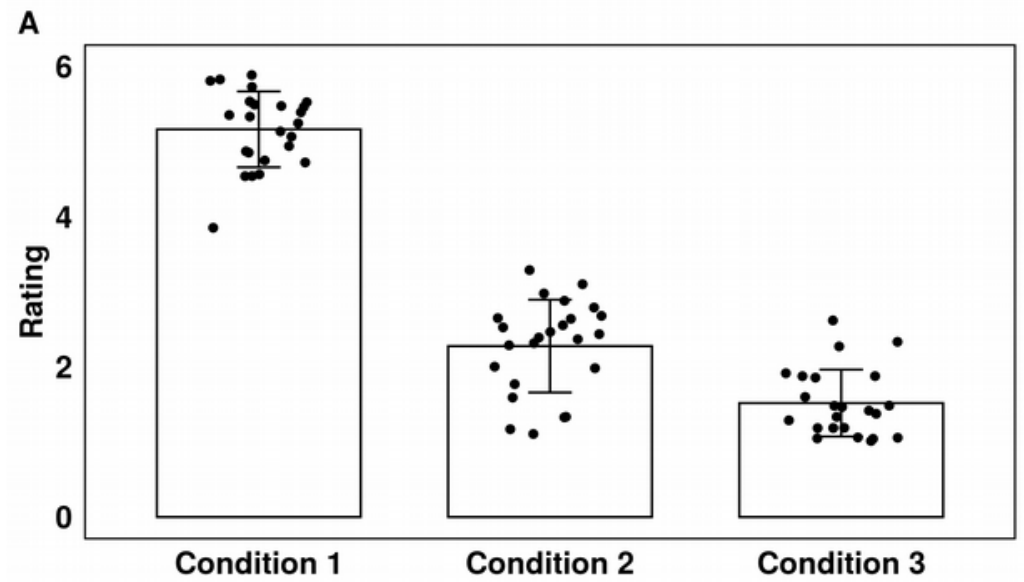

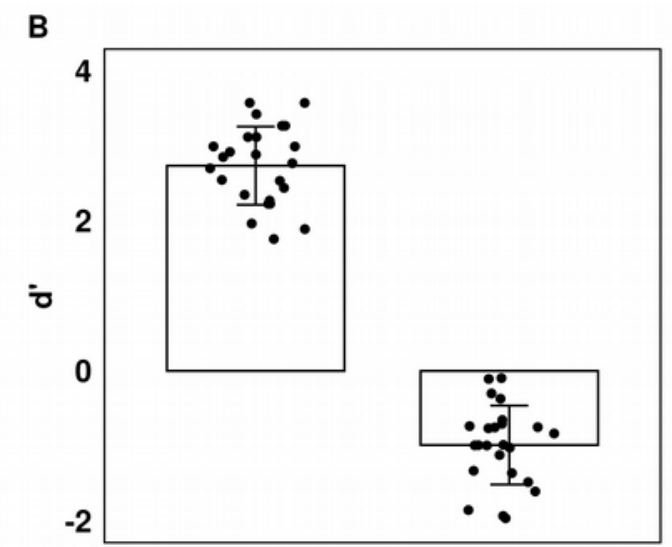

Attestedness Well-formedness

Figure 2: (A) Mean ratings given by participants in each condition. Condition 1: Non-words with attested and well-formed onsets, Condition 2: Non-words with unattested and well-formed onsets, Condition 3: Non-words with unattested and ill-formed onsets. (B) Mean d' for attestedness and well-formedness. Each point represents one participant. Error bars represent standard errors.

\section{Discussion}

We examined the sensitivity of speakers to the lexical attestedness and phonological well-formedness (with respect to the SSP) of CC onsets in French using a non-word acceptability task. We used the signal detection theory, which is a useful technique to analyze acceptability judgments in language research (Huang \& Ferreira, 2020). The results showed that participants were sensitive to attestedness. This was reflected by d'values much larger than 0. Participants were also able to distinguish non-words with unattested well-formed onsets from those with unattested ill-formed onsets. This was reflected by d' values which were different from 0 . The results 
are thus consistent with previous findings on sonority projection effects (e.g. Daland et al., 2011), showing that participants rate non-words with well-formed onsets as more acceptable, irrespective of their attestedness. Since the condition with non-words whose onsets were unattested and well-formed was used as the reference, (see Figure 1 and Equations 1 and 2), a positive and negative d' means that the responses were respectively greater and smaller than the responses to non-words with unattested and well-formed onsets. Thus, the sensitivity to each manipulated factor (i.e. attestedness and well-formedness) corresponds to the absolute values of $d$ '.

The main aim of the study was to investigate the interplay between the processing of attestedness and well-formedness. Our results on d' show that the sensitivity of participants to attestedness was higher than their sensitivity to well-formedness (absolute mean d': 2.73 vs. 0.99). This shows that participants perceived non-words whose onset differed regarding attestedness to be more distinct than those whose onset differed regarding the well-formedness of their onset. Indirectly, this could be consistent with the view that participants' knowledge about well-formedness is not based on statistical properties extracted from the lexicon (Berent et al., 2007, for a review see Berent et al., 2017) and that attestedness and well-formedness are perceived differently.

This interpretation is consistent with studies that observed brain responses to well-formedness that were independent of attestedness. In an fMRI study in English, Deschamps, Baum, and Gracco (2015) examined brain responses to the presentation of non-words beginning with $\mathrm{CC}$ clusters that had different sonority rankings (rising vs. plateau/less rising). They observed that the activity of the posterior part of the left inferior frontal gyrus was modulated by the sonority ranking of the onsets in the reading task. In an EEG study of coda clusters in German, Ulbrich et al. (2016) examined the effect of well-formedness on word learning. They investigated the relative ease or difficulty with which novel words containing these clusters could be learned when the clusters violated or respected the SSP. Participants performed a matching task between auditory non- 
words and pictures after a learning phase. The authors also investigated the interaction between wellformedness and the frequency of clusters in the lexicon using attested and unattested clusters. The results showed distinct but interacting responses to well-formedness and attestedness (see also Wiese, Orzechowska, Alday, \& Ulbrich (2017) for a study of Polish). In a passive-listening EEG study in English, White and Chiu (2017) also observed distinct responses to the attestedness of CC onsets and their well-formedness. In line with these findings, our results support the view that speakers rely on two sources of knowledge, i.e. formal phonological rules such as the SSP and statistical properties extracted from the lexicon, when processing novel forms in their language, with the sensitivity to attestedness being higher that the sensitivity to well-formedness.

In the current study, we focused on syllable frequency and did not consider the frequency of features when constructing our linguistic material. Thus, the unattested well-formed clusters we used may still have been more attested than unattested ill-formed clusters in terms of feature frequency. A model that can extract and generalize featural cues from the lexicon (e.g. Phonotactic Learner, Hayes \& Wilson, 2008) can also predict sonority projection effects by using only lexical statistics (Daland et al., 2011). In future work, it will be important to investigate whether similar results are obtained without adding any assumption about the SSP in this model. Importantly, Hayes and White (2013) using a modeling approach observed that while some constraints could be extracted from the lexicon, others could not. A similar approach could be used to establish whether the observed difference in the current study between sensitivity to lexical attestedness and to phonological well-formedness is due to lexical knowledge (e.g. issues such as differences in terms of the frequency of features), or whether it reflects the fact that both lexical attestedness and phonological wellformedness are used by speakers, but to a different extent.

Our study had some limitations. First, we used printed materials. To minimize the impact of the issues related to grapheme-phoneme correspondence and written language processing, it would be interesting to replicate the study using auditory stimuli. However, it is unlikely that the observed pattern was dependent on 
the modality (auditory vs. printed), as previous studies showed that sonority projection effects extend to printed materials because the underlying mechanisms are related to phonological knowledge (Berent \& Lennertz, 2010; Daland et al., 2011). The second limitation concerns the behavioral task we used. Although the non-word acceptability task has been widely used (e.g. Greenberg \& Jenkins, 1964; Albright, 2009; Daland et al., 2011; Hayes \& White, 2013), such tasks might not fully engage the phonological system of speakers. For instance, in a non-word generation task, Bucci et al. (2019) observed wide variability between participants and suggested that some participants may not have considered the task as a linguistic task but rather as a nonlinguistic game. While this possibility cannot be ruled out, note that the variabilities between participants regarding the sensitivity to attestedness and well-formedness were small (see Figure 2), so our findings were likely unaffected by this issue. The third limitation concerns the sonority scale that we used to create our stimuli. In fact, we used Steriade's (1982) sonority hierarchy, with fricatives more sonorous than stops as for instance in the study of Maïonchi-Pino et al. (2015) in French. However, a coarse-grained scale as in Clements (1988) (obstruents < nasals < liquids < glides < vowels) seems to be more consensual (e.g. Daland et al., 2011). Although most of our clusters were also consistent with the latter scale (see Appendix A), few of them such as $f k$ were not. Thus, it would be interesting to replicate our results with a linguistic material created using a less fined-grained scale.

Overall, using an acceptability judgment task as in Daland et al. (2011), our findings show the existence of the sonority projection effect in French. They also show that French speakers are more sensitive to lexical attestedness than to phonological well-formedness (defined as sonority rising vs. sonority falling onsets). This suggests that knowledge of phonological constraints regarding the SSP is used differently from that concerning the statistical properties of the lexicon. In addition, our study shows that the signal detection theory approach is useful to analyze data obtained on sonority projection effects, thus extending the models discussed in Huang 
and Ferreira (2020). Future studies should investigate whether and how our experimental results could potentially be obtained without any assumption (or a moderate assumption, e.g. Hayes, 2011) about the SSP.

\section{Declaration of conflicting interest}

The authors have no conflicts of interest to declare.

\section{Acknowledgments}

We thank the participants in this study. We are grateful to Marine Guiborel, Roxane Kamphuis, Laurent Ott, and Laetitia Zmuda for their help in preparing the stimuli and/or collecting data. 


\section{References}

Albright, A. (2009). Feature-based generalisation as a source of gradient acceptability*. Phonology, 26(1), 9-41. https://doi.org/10.1017/S0952675709001705

Bárkányi, Z. (2011). Gradient phonotactic acceptability a case study from Slovak. Acta Linguistica Hungarica, 58(4), 353-390.

Berent, I. (2017). On the origins of phonology. Current Directions in Psychological Science, 26(2), 132-139. https://doi.org/10.1177/0963721416688891

Berent, I., \& Lennertz, T. (2010). Universal constraints on the sound structure of language: Phonological or acoustic? Journal of Experimental Psychology: Human perception and performance, 36(1), 212. https://doi.org/10.1037/a0017638

Berent, I., Steriade, D., Lennertz, T., \& Vaknin, V. (2007). What we know about what we have never heard: Evidence from perceptual illusions. Cognition, 104(3), 591-630. https://doi.org/10.1016/j.cognition.2006.05.015

Bucci, J., Lorusso, P., Gerber, S., Grimaldi, M., \& Schwartz, J.-L. (2019). Assessing the Representation of Phonological Rules by a Production Study of Non-Words in Coratino. Phonetica, 1-24. https://doi.org/10.1159/000504452

Clements, G. N. (1990). The role of the sonority cycle in core syllabification. In J. Kingston \& M. Beckman (eds.), Papers in laboratory phonology 1: between the grammar and physics of speech. Cambridge, Cambridge University Press: 283-333. https://doi.org/10.1017/CBO9780511627736.017

Daland, R., Hayes, B., White, J., Garellek, M., Davis, A., \& Norrmann, I. (2011). Explaining sonority projection effects. Phonology, 28(2), 197-234. https://doi.org/10.1017/S0952675711000145

Deschamps, I., Baum, S. R., \& Gracco, V. L. (2015). Phonological processing in speech perception: What do sonority differences tell us? Brain and Language, 149, 77-83. https://doi.org/10.1016/j.bandl.2015.06.008 
Greenberg, J. H. 1978. Some Generalizations Concerning Initial and Final Consonant Clusters. In J. H. Greenberg, C. A. Ferguson \& E. A. Moravscik (eds.), Universals in Human Language, vol. 2: Phonology. Stanford (CA), Stanford University Press: 243-279. https://doi.org/10.1515/ling.1965.3.18.5

Greenberg, J. H., \& Jenkins, J. J. (1964). Studies in the Psychological Correlates of the Sound System of American English. WORD, 20(2), 157-177. https://doi.org/10.1080/00437956.1964.11659816

Hayes, B. (2011) Interpreting sonority-projection experiments: the role of phonotactic modeling. Proceedings of the International Congress of Phonetic Sciences, Hong Kong, pp. 835-838.

Hayes, B., \& White, J. (2013). Phonological Naturalness and Phonotactic Learning. Linguistic Inquiry, 44(1), 45-75. https://doi.org/10.1162/LING_a_00119

Hayes, B., \& Wilson, C. (2008). A Maximum Entropy Model of Phonotactics and Phonotactic Learning. Linguistic Inquiry, 39(3), 379-440. https://doi.org/10.1162/ling.2008.39.3.379

Henke, E., E. Kaisse \& R. Wright (2012). Is the sonority sequencing principle an epiphenomenon? In S. Parker S (ed.), The sonority controversy. Berlin, Mouton de Gruyter: 65-100. https://doi.org/10.1515/9783110261523.65

Huang, Y., \& Ferreira, F. (2020). The application of signal detection theory to acceptability judgments. Frontiers in Psychology, 11.

Jarosz, G. (2017). Defying the stimulus: acquisition of complex onsets in Polish. Phonology, 34(2), 269-298.

Jozefowiez, J., Gaudichon, C., Mekkass, F., \& Machado, A. (2018). Log versus linear timing in human temporal bisection: A signal detection theory study. Journal of Experimental Psychology: Animal Learning and Cognition, 44(4), 396-408. https://doi.org/10.1037/xan0000184

Macmillan, N. A., \& Creelman, C. D. (1991). Detection theory: A user's guide. New York, NY: Cambridge University Press.

Macoir, J., Gauthier, C., Jean, C., \& Potvin, O. (2016). BECLA, a new assessment battery for acquired deficits of language: Normative data from Quebec-French healthy younger and older adults. Journal of the Neurological Sciences, 361, 220-228. https://doi.org/10.1016/i.jns.2016.01.004 
Maïonchi-Pino, N., Taki, Y., Magnan, A., Yokoyama, S., Écalle, J., Takahashi, K., ... \& Kawashima, R. (2015). Sonority-related markedness drives the misperception of unattested onset clusters in French listeners. LAnnee psychologique, 115(2), 197-222.

Majerus, S., van der Kaa, M.-A., Renard, C., Van der Linden, M., \& Poncelet, M. (2005). Treating verbal shortterm memory deficits by increasing the duration of temporary phonological representations: A case study. Brain and Language, 95(1), 174-175. https://doi.org/10.1016/j.bandl.2005.07.094

New, B., Pallier, C., Brysbaert, M., \& Ferrand, L. (2004). Lexique 2: A new French lexical database. Behavior Research Methods, Instruments, \& Computers, 36(3), 516-524. https://doi.org/10.3758/BF03195598

Parker, S. (2017). Sounding out Sonority. Language and Linguistics Compass, 11(9), n/a-n/a. https://doi.org/10.1111//nc3.12248

Sato, M., Grabski, K., Glenberg, A. M., Brisebois, A., Basirat, A., Menard, L., \& Cattaneo, L. (2011). Articulatory bias in speech categorization: Evidence from use-induced motor plasticity. Cortex, 47(8), 1001-1003.

Sievers, E. (1876). Grundzüge der lautphysiologie zur einführung in das studium der lautlehre der indogermanischen sprachen. Breitkopf und Hartel.

Steriade, D. (1982). Greek prosodies and the nature of syllabification. Ph.D. dissertation, MIT. Published 1990, New York, Garland.

Ulbrich, C., Alday, P. M., Knaus, J., Orzechowska, P., \& Wiese, R. (2016). The role of phonotactic principles in language processing. Language, Cognition and Neuroscience, 31(5), 662-682. https://doi.org/10.1080/23273798.2015.1136427

van de Vijver, R., \& Baer-Henney, D. (2012). Sonority intuitions are provided by the lexicon. In S. Parker(Ed.),The sonority controversy(pp. 195-218). Berlin: Walter de Gruyter.

White, J., \& Chiu, F. (2017). Disentangling phonological well-formedness and attestedness: An ERP study of onset clusters in English. Acta Linguistica Academica, 64(4), 513-537. https://doi.org/10.1556/2062.2017.64.4.2 
Wiese, R., Orzechowska, P., Alday, P. M., \& Ulbrich, C. (2017). Structural Principles or Frequency of Use? An ERP Experiment on the Learnability of Consonant Clusters. Frontiers in Psychology, 7. https://doi.org/10.3389/fpsyg.2016.02005 


\section{Appendix A}

Onsets and tails used in study to create 144 CCVC non-words (48 per condition).

\begin{tabular}{|c|c|}
\hline Attested and well-formed clusters & pl, pr, tr, kl, kr, bl, br, dr, gl, gr, fl, fr, sl \\
\hline Unattested and well-formed clusters & pv, pm, tv, tm, tn, tl, kv, km, kn, bn, dm, dl, gm \\
\hline Unattested and ill-formed clusters & fk, ft, vg, vp, zt, ms, nd, lp, lk, ls, rt, rb, rf \\
\hline Tails & ic, ive, ine, ir, uc, use, ule, ur, age, ane, al, ar \\
\hline
\end{tabular}

\section{Appendix B}

Properties of non-words used in study, extracted from Lexique database (New et al., 2004), averaged per Condition. Frequencies are in terms of occurrences per million.

\begin{tabular}{|l|c|c|c|}
\hline & Attested and & Unattested and & Unattested and ill- \\
& well-formed & well-formed & formed \\
\hline CC sonority profile & condition & condition & condition \\
\hline CC type frequency & 4 & 3.75 & -3.23 \\
\hline CC token frequency & 2163.65 & 2.17 & 1.15 \\
\hline CC type frequency in onset position & 5921.20 & 0.92 & 0.21 \\
\hline CC token Frequency in onset position & 867.79 & 0.52 & 1.15 \\
\hline Phonological neighbors & 2674.77 & 0.04 & 0.21 \\
\hline Orthographic neighbors & 11.33 & 3.96 & 3.96 \\
\hline
\end{tabular}

